\title{
ARTICLE OPEN Prophylactic effects of isomaltodextrin in a Balb/c mouse model of egg allergy
}

Yoshinori Mine ${ }^{1 *}$, Yan Jin ${ }^{1,3}$, Hua Zhang ${ }^{1}$, Prithy Rupa ${ }^{1}$, Kaustav Majumder ${ }^{1,4}$, Takeo Sakurai ${ }^{2}$, Yoshifumi Taniguchi ${ }^{2}$, Ryodai Takagaki ${ }^{2}$, Hikaru Watanabe ${ }^{2}$ and Hitoshi Mitsuzumi ${ }^{2}$

The aim of this study was to evaluate the potential effects of isomaltodextrin (IMD), a dietary saccharide polymer derived from enzymatically produced from starch, on the ability to alter immune response (IR) bias to hen egg ovalbumin (Ova) induced allergic inflammation in mice. Groups of Balb/c mice were pre-treated with various doses of IMD in drinking water (1.0, 2.5, and 5.0\% w/v) for 6 weeks and subsequently sensitized to the Ova together with continuous administration of IMD. To evaluate changes in immune response bias, immunoglobulin isotype-associated antibody activity, concentrations of type 1 and 2 cytokines and the percentage of T-regulatory cells (T-regs) in blood were measured. Clinical signs of allergy were assessed after oral challenge with Ova. Treatment with IMD did not significantly alter the frequency of clinical signs, however there was a trend in the overall reduction of clinical signs. Effect on IR bias was observed in the treatment groups as reflected by reduction in a type 1-biased phenotype as evident by decrease in isotype-specific $\lg \mathrm{E}$, IgG and increase in IL-12 cytokine production and a high proportion of Tregs. This study revealed that IMD could be a useful prophylactic candidate for alteration of allergic IR bias in mice and an immunestimulator for reducing egg induced allergic reactions.

npj Science of Food (2019)3:23 ; https://doi.org/10.1038/s41538-019-0057-5

\section{INTRODUCTION}

Foods that may cause allergic reactions are particularly insidious. Food allergy, an adverse immunologic reaction to food, affects $6-8 \%$ of children and $4 \%$ of adults in North America. ${ }^{1,2}$ The prevalence of food allergy in children (aged 0 to 17 years) has slowly increased in the USA, from $3.4 \%$ in $1997-1999$ to $5.1 \%$ in 2009-2011, associated the major foods of milk, eggs, fish, crustacean shellfish, wheat, soy, peanuts, and tree nuts. ${ }^{3}$ It has been reported that $7.5 \%$ of Canadians-7.7\% of adults and $6.9 \%$ of children under 18 years of age having at least one food allergy, most commonly associated with peanut, tree nut, fish, shellfish, sesame, milk, egg, wheat, and soy. ${ }^{4}$ Both the prevalence and severity of food allergies have increased in past few decades., ${ }^{5,6}$ Egg, milk, and peanut together contribute to nearly $90 \%$ of the food allergies. ${ }^{7}$ A recent study by FAAN (Food Allergy and Anaphylaxis Network) and FARP (Food Allergy Research and Resource Program) reported that over 12 million Americans suffer from food allergies. ${ }^{8,9}$ This indicates that food allergies are occurring at an alarmingly high rate, pressing the need for efficient preventive measures. The prevalence of childhood food allergy and the duration of these allergies, particularly those considered to be transient, like egg and milk allergy continues to increase. Methods to evaluate the potential allergenicity of foods are limited and research in developing assessment tools is needed to investigate factors for allergenic susceptibility and to improve on valid animal models for food allergy. Food allergies not only affect the susceptible individuals but also have an impact on food industries especially with the labeling laws and manufacturing practices.

To reduce the risk of food allergy, anti-lgE therapy, SOTI (specific oral tolerance induction), PIT (peptide-based immunotherapy), DNA-based immunotherapy, genetically engineered egg allergens and off late probiotics have come up with promising results but needs an in-depth study. ${ }^{10}$ Also, currently available therapeutics (anti-histamine, epinephrine, and steroids) provide only symptomatic relief. Due to the complex nature of allergic disease, standard treatments are limited to allergen avoidance, nutritional support, and immediate access to emergency medication. Immunoglobulin E (IgE)-mediated immune response to egg proteins in food can be life-threatening, leading to responses ranging from repeated scratching, diarrhea, vomiting, and rarely resulting in death. ${ }^{11}$ Mainly glycoproteins from egg white have been identified as food allergens. Among all the glycoproteins, egg white Ovalbumin (Ova), and ovomucoid (Ovm) are classified as dominant allergens and play a crucial role in the egg-induced allergenic response. ${ }^{12}$ Ovm is heat resistant and resistant to digestive enzymes whereas Ova is easily digested and absorbed in the gut. ${ }^{13}$

Most individuals who suffer from food allergy are polysensitized and therefore allergic to more than one food. ${ }^{14}$ Allergen specific immunotherapies would not be beneficial in this case and hence the use of allergen-nonspecific therapy could circumvent this problem by enabling change in the host immune response (IR), which ideally would induce tolerance toward all food allergens. Polysaccharides have been shown to act as prebiotics with IR modulating properties for the prevention of food allergies. ${ }^{15}$

Prebiotics are defined as "a substrate that is selectively utilized by host microorganisms conferring a health benefit" ${ }^{16}$ The criteria that are classically met by dietary prebiotics are: resistance to acidic gastric $\mathrm{pH}$ and mammalian digestive enzymes, as well as high absorption yield through the gastrointestinal (GI) tract. Maltodextrins are branched polysaccharides composed by a maximum of seventeen chains of dextrose molecules, linked with alpha-glycosidic bonds consisting of a $(1 \rightarrow 4)$ and $a(1 \rightarrow 6)$ linked

\footnotetext{
${ }^{1}$ Department of Food Science, University of Guelph, Guelph, ON N1G2W1, Canada. ${ }^{2}$ R\&D Center, Hayashibara CO., LTD., 675-1 Fujisaki, Naka-ku, Okayama 702-8006, Japan; ${ }^{3}$ Present address: College of Food Engineering and Biotechnology, Tianjin University of Science and Technology, 300457 Tianjin, People's Republic of China and ${ }^{4}$ Present address: Department of Food Science and Technology, University of Nebraska-Lincoln, Lincoln, NE 68588-6205, USA. *email: ymine@uoguelph.ca
} 
D-glucose units. Isomaltodextrin (IMD) (GI resistant maltodextrin) is a highly branched soluble a-glucan with a relatively low degree of polymerization. ${ }^{17}$ This glucan partially escapes digestion in the small intestine and may undergo fermentation by bacteria in the large intestine, and therefore, it could play a role as a prebiotic. ${ }^{17,18}$ It is considered as generally recognized as safe (GRAS) by the US Food and Drug Administration (FDA). Fructooligosaccharides (FOSs) occur naturally in a wide variety of foods. In fact, Americans consume $\sim 2.5 \mathrm{~g}$ of inulin and oligofructose daily (range of 1-4 g), mostly from wheat and onions. ${ }^{19}$ In terms of safety, FOSs cause few adverse effects, and the adverse effects are minor in nature. ${ }^{20}$

Maltodextrin and its enzymatically modified forms have been investigated for different biomedical properties. IMD was digested partially only by small intestinal mucosal membrane in healthy subjects and reduced the glucose. ${ }^{21,22}$ The efficacy of IMD in the treatment of intestinal inflammation was also investigated in a mouse model of colitis and resulted in a significant reduction in the expression of pro-inflammatory mediators TNF- $a$ and IL-8, as well as pattern recognition receptor TLR4. ${ }^{23}$ However, the effect of IMD on allergenic response has not been elucidated yet. Therefore, the primary objective of the current project is to evaluate the effect of IMD on Ova-induced allergenic response in mice. It was hypothesized that IMD could alter the IR phenotype and expression of allergy in mice sensitized to the egg white allergen Ova.

\section{RESULTS}

Clinical scores and allergenic response of mice

On the final experimental day, allergic reaction was induced into the mice by oral gavage by $20 \mathrm{mg}$ of Ova and then the mice were observed for next 30 min for clinical scoring. Figure 1 illustrated the clinical signs observed in different groups of mice. Clinical scores were assigned and the total scores for each animal were obtained by adding scores for the individual symptoms of scratching, sneezing, isolation, diarrhea and lethargy and respiratory difficulty. Post challenge, animals in the positive control group developed statistically significant clinical scores (3.889 \pm $0.254)$ of allergies as compared to the negative control (1.182 \pm $0.296)(p<0.0001)$. Prevention groups with IMD (Low-L, Medium$M$, and High-H) (L: $3.500 \pm 0.267$, M: $3.200 \pm 0.291, \mathrm{H}: 3.417 \pm 0.288$ ) did not reach any statistical significance compared to the positive control group, however a trend towards a decrease in clinical scores was observed with the treatment groups compared to the positive control.

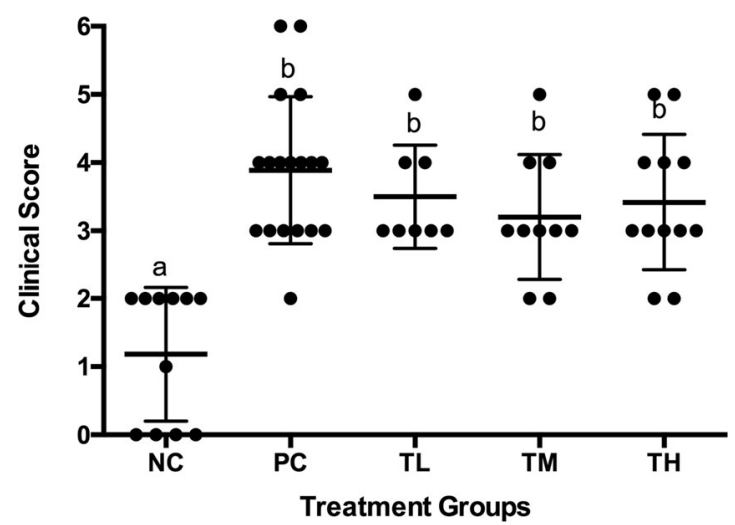

Fig. 1 Clinical scores for individual mice. Total clinical scores for each mice post-Ova challenge were calculated. Average scores assigned by four independent observers in a blinded fashion are represented. Different letters indicate statistically significant differences
Histamine and mast cell protease concentration

Histamine and mast cell protease concentration were evaluated as a measure for the mast cell activation and degranulation. Both histamine and mast cell protease concentration were increased in the positive control (Histamines: $70.598 \pm 7.043 \mathrm{ng} / \mathrm{mL}$, MMCPT-1: $1354.313 \pm 196.402 \mathrm{ng} / \mathrm{mL}$ ) significantly compared to the negative control group (Histamine: $22.957 \pm 2.053 \mathrm{ng} / \mathrm{mL}, \quad P<0.0001$, MMCPT-1: $312.712 \pm 35.882 \mathrm{ng} / \mathrm{mL}, P<0.001)$. Prevention groups of low, medium, and high-dose IMD showed statistical significant decrease in histamine (L: $31.835 \pm 4.957 \mathrm{ng} / \mathrm{mL}, P<0.001 ; \mathrm{M}$ : $24.297 \pm 5.452 \mathrm{ng} / \mathrm{mL}, \quad P<0.0001 ; \quad \mathrm{H}: 23.251 \pm 7.877 \mathrm{ng} / \mathrm{mL}, \quad P<$ 0.0001 ) as compared to positive control group. Prevention groups with low and high-dose IMD showed statistical significant decrease in mast cell protease (L: $682.140 \pm 56.801 \mathrm{ng} / \mathrm{mL}, P<$ $0.05 ; \mathrm{H}: 641.263 \pm 36.807 \mathrm{ng} / \mathrm{mL}, \quad P<0.05)$ compared to positive control group. Prevention group of medium-dose IMD (717.737 \pm $103.845 \mathrm{ng} / \mathrm{mL}$ ) did not show statistical significant decrease in mast cell protease compared to positive control group (Fig. $2, p<$ $0.05)$. However, there was no significant difference observed with the IMD treated groups $(p>0.05)$ with both histamine and mast cell protease concentration as measured.

Antibody concentrations in plasma and feces

Specific and total antibody levels were measured in the blood plasma. There was a significant reduction of total lgG, total lgG1, and IgG2a in all the three prevention groups (IgG L: $478.745 \pm$

\section{Histamine}
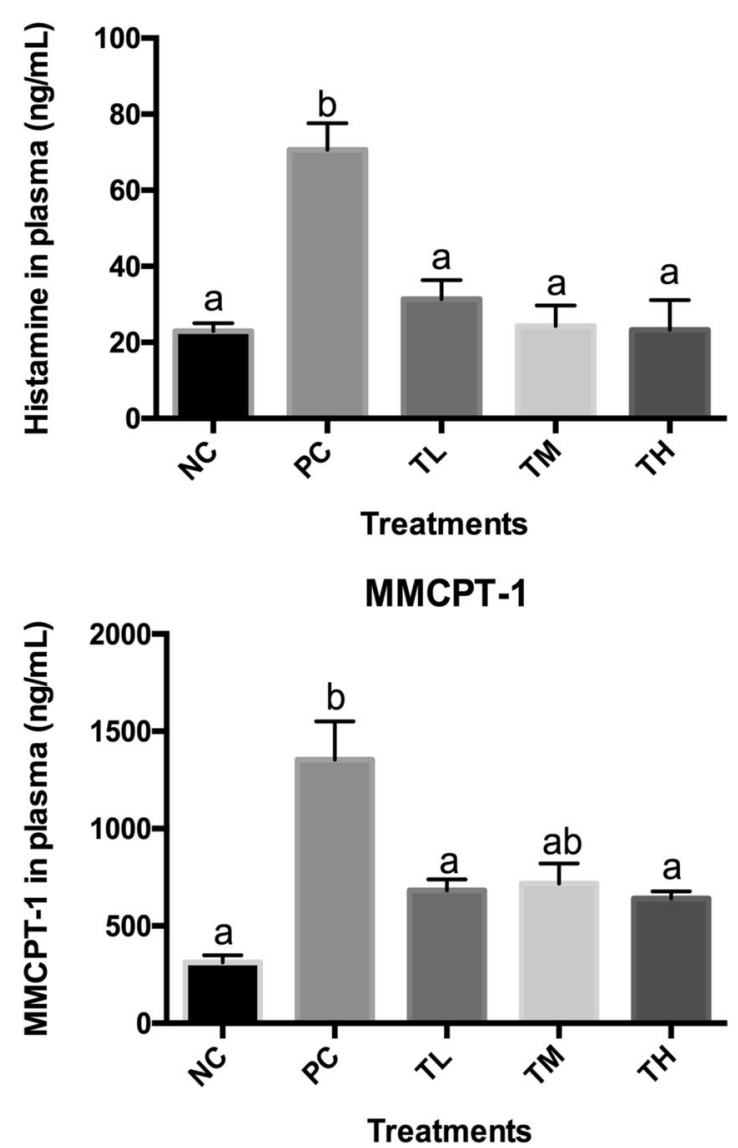

Fig. 2 Serum histamine and mouse mast cell protease concentration. Data for serum histamine and MMCP concentration are represented as mean \pm standard deviation ( $n=6$ pooled sera). Different letters indicate statistically significant differences $(p<$ 0.05 ) between groups of mice 
$\lg G$

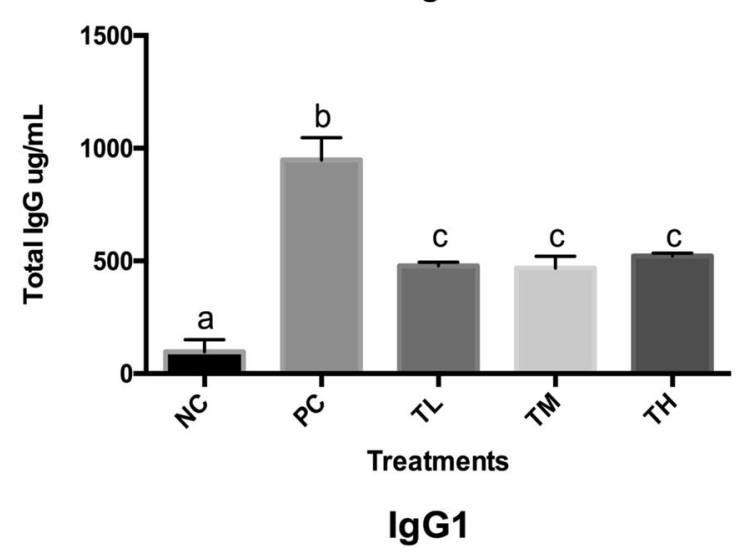

$\lg G 2 a$

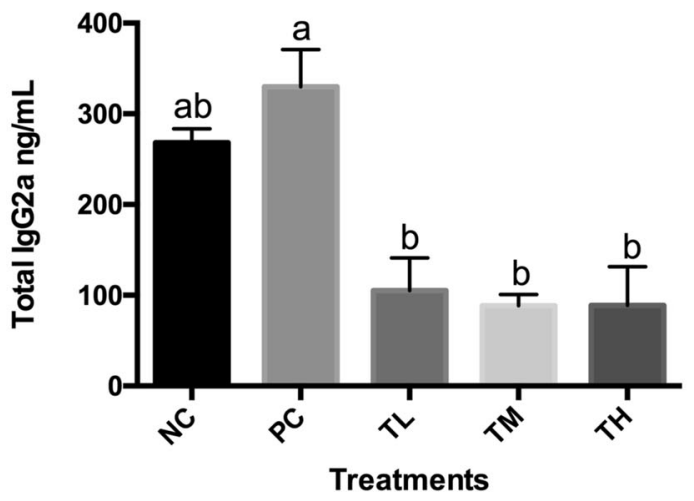

Total lgE

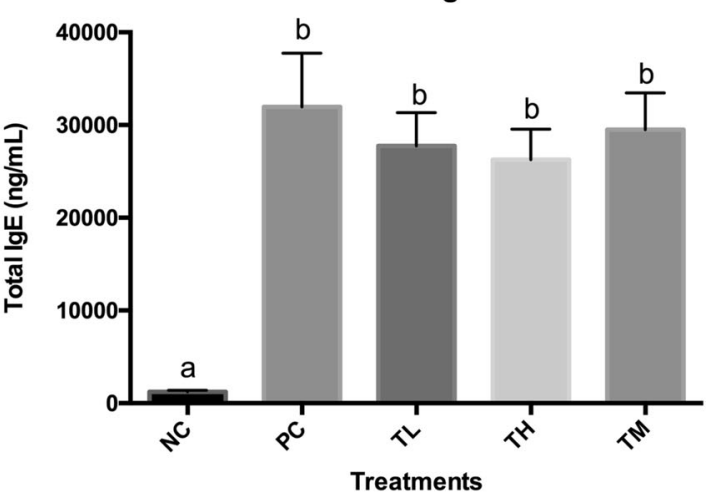

Fig. 3 Total IgG (whole molecule), IgE, IgG1, and IgG2 related antibody activity. Rabbit anti-mouse IgG conjugated to alkaline phosphatase

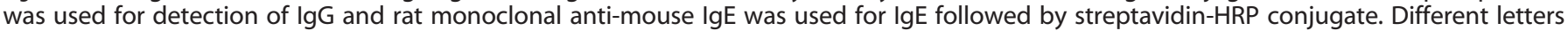
indicate statistically significant differences at $p<0.05$

$6.409 \mathrm{ng} / \mathrm{mL}, P<0.0001 \mathrm{M}: 467.624 \pm 21.669 \mathrm{ng} / \mathrm{mL}, P<0.0001, \mathrm{H}:$ $522.438 \pm 4.696 \mathrm{ng} / \mathrm{mL}, P<0.0001$ vs. PC: $948.307 \pm 28.469 \mathrm{ng} / \mathrm{mL}$; lgG1 L: $\quad 4326.344 \pm 332.243 \mathrm{pg} / \mathrm{mL}, \quad P<0.001 \mathrm{M}: \quad 4106.144 \pm$ $255.851 \mathrm{pg} / \mathrm{mL}, P<0.0001, \mathrm{H}: 4766.541 \pm 236.048 \mathrm{pg} / \mathrm{mL}, P<0.05$ vs. PC: $5627.638 \pm 101.712 \mathrm{pg} / \mathrm{mL}$; IgG2a L: $105.034 \pm 36.056 \mathrm{ng} /$ $\mathrm{mL}, P<0.01, \mathrm{M}: 88.636 \pm 12.297 \mathrm{ng} / \mathrm{mL}, P<0.01, \mathrm{H}: 105.825 \pm$ $48.216 \mathrm{ng} / \mathrm{mL}, P<0.01$ vs. PC: $329.994 \pm 40.947 \mathrm{ng} / \mathrm{mL}$ ) (Fig. 3). The total IgE showed no statistical significant difference in all three prevention groups (L: $27748.230 \pm 3598.297 \mathrm{ng} / \mathrm{mL}$, M:29495.240 $\pm 3975.996 \mathrm{ng} / \mathrm{mL}, \quad H: 26272.100 \pm 3269.893 \mathrm{ng} / \mathrm{mL}$ ) compared to positive control group (31957.490 $\pm 5805.165 \mathrm{ng} /$ $\mathrm{mL}$ ). Specific lgG2a was significantly reduced in the high dose IMD group $(1.741 \pm 0.043)$ compared to positive control group (1.983 \pm $0.033, P<0.01)$. Specific lgG1(L: $1.324 \pm 0.032, P<0.0001, M$ : $1.230 \pm 0.034, P<0.0001 \mathrm{H}: 1.225 \pm 0.036, P<0.0001)$ and specific $\operatorname{lgE} \quad(\mathrm{L}: \quad 0.941 \pm 0.121, P<0.0001 \mathrm{M}: 0.908 \pm 0.104, P<0.0001$, $\mathrm{H}: 0.868 \pm 0.095, P<0.0001)$ levels decreased significantly after IMD pre-treatment in all three doses as compared to the positive control group (specific lgG1:1.924 \pm 0.032 ; specific lgE: $1.504 \pm$ 0.089) (Fig. 4). The specific IgA level was measured from the total protein extracted from the mice feces. The specific IgA showed no significant change in the prevention groups as compared to the positive control group (Fig. 5).

\section{Cytokine concentration}

Cytokine expression in splenocyte was measured from the cultured spleen cells. No statistically significant difference was observed in the prevention groups as compared to the positive and negative control groups with IL-4, IL-10, and IL-17 concentrations (Fig. 6) $(p>0.05)$. Although the clear significant was not observed due to high variable of ranges, the trend of decreasing IL-4, while increasing IL-10 and IL-17 was observed. Prevention groups of medium dose $(35.440 \pm 3.908 \mathrm{pg} / \mathrm{mL}, P<$ $0.01)$ and high dose $(35.475 \pm 2.054 \mathrm{pg} / \mathrm{mL}, P<0.05)$ of IMD showed statistical significantly decreased of TGF- $\beta$ as compared to positive control group $(58.957 \pm 4.054 \mathrm{pg} / \mathrm{mL})$. High-dose prevention group $(3.226 \pm 1.346 \mathrm{ng} / \mathrm{mL}, \quad P<0.05)$ significantly decreased IFN- $\gamma$ compared to positive control group (19.546 \pm $3.071 \mathrm{ng} / \mathrm{mL}$ ). IL-12 concentration was significantly increased in a dose-dependent manner after IMD treatment with the TM $\quad(476.945 \pm 119.006 \mathrm{pg} / \mathrm{mL}, \quad P<0.05)$ and TH $(589.460 \pm$ $90.797 \mathrm{pg} / \mathrm{mL}, \quad P<0.01)$ compared to $P C$ group $(13.654 \pm$ $2.507 \mathrm{pg} / \mathrm{mL}$ ) (Fig. 6).

Percentage of Treg cells

The flow cytometry analysis showed that the percentage of Foxp3 and CD25+ cells significantly increased in all the three prevention groups (L: $51.700 \pm 3.758, \mathrm{M}: 50.717 \pm 7.349, \mathrm{H}: 49.400 \pm 9.489$ ) (Fig. 7) as compared to those in the positive control group $(19.600 \pm 5.424)(p<0.05)$.

\section{DISCUSSION}

Food allergy is an immunologically adverse reaction caused by foods. It is an IgE-dependent type I hypersensitivity reaction due to the imbalance of Th1/Th2. A food allergic murine model sensitized by intraperitoneally followed by the orally challenge of anti-allergic bioactive compounds was established in our past 

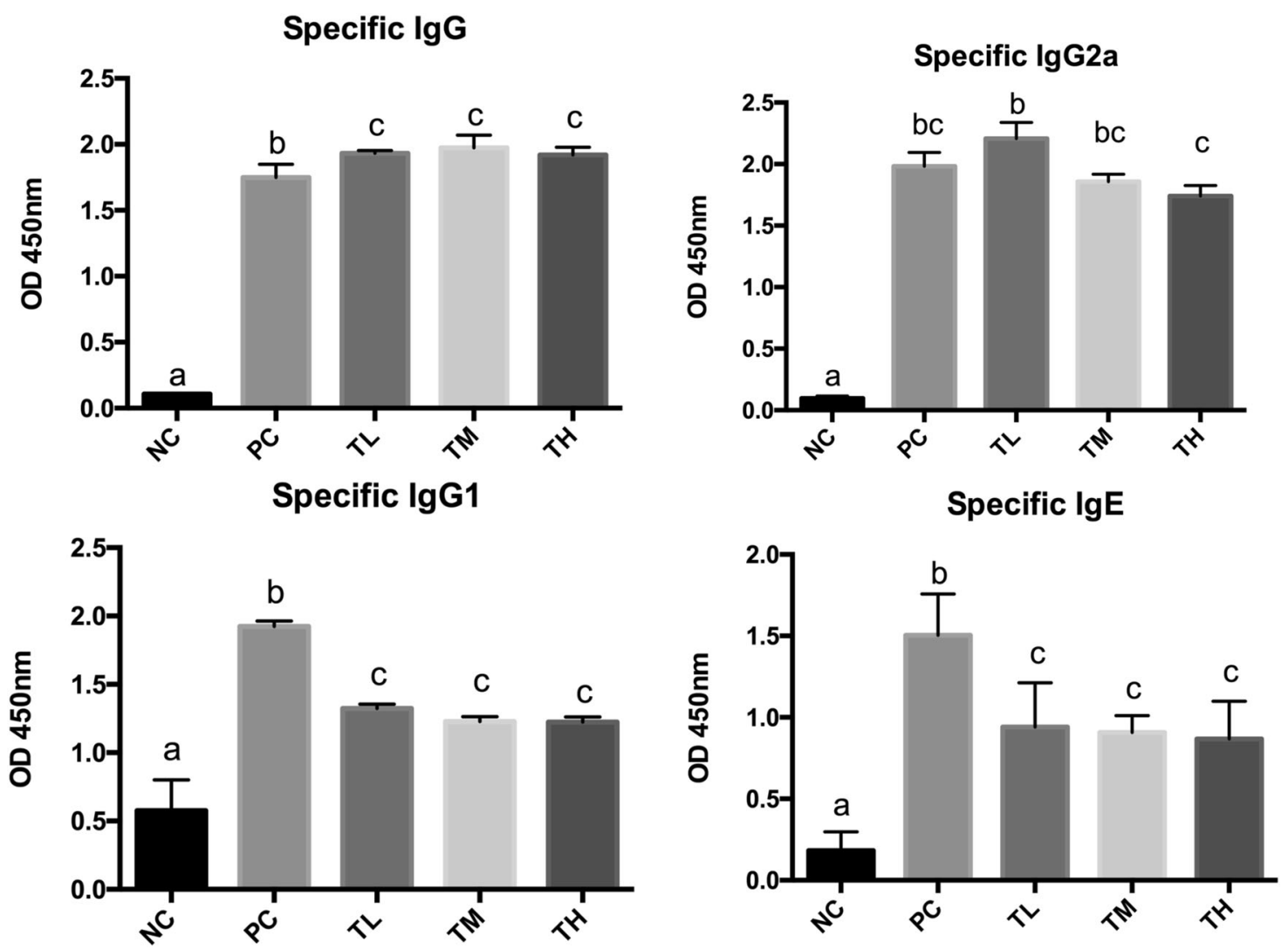

Fig. 4 Ova-specific serum IgG, IgG1, IgG2, and IgE-related antibody activity. Rat monoclonal anti-mouse, IgG, IgG1, IgG2a, and IgE were used followed by streptavidin-HRP conjugate. Different letters indicate statistically significant differences at $p<0.05$

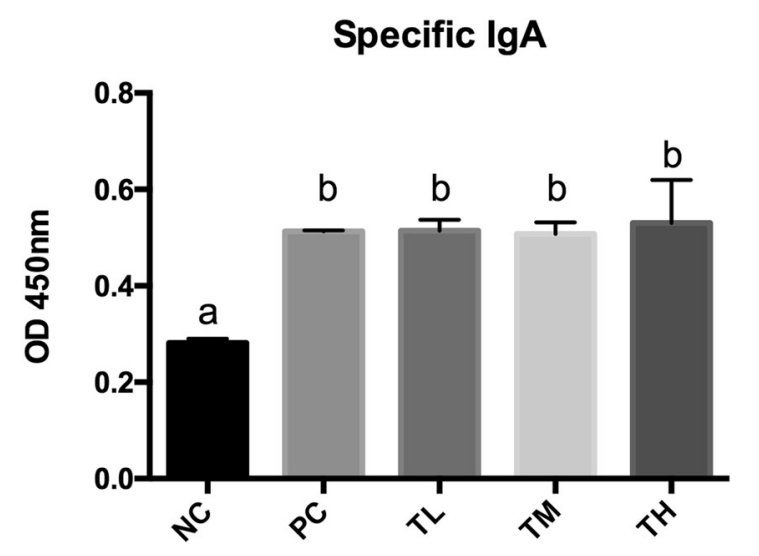

Fig. 5 Ova-specific IgA was detected from pooled fecal samples collected from weeks 9-11 using biotinylated rat monoclonal antimouse IgA antibody followed by avidin-HRP conjugate. Different letters indicate statistically significant differences

study. ${ }^{24}$ The present data suggested that pre-treatment with IMD (for 6 weeks) and continuous administration of IMD (week 7-11) during Ova sensitization influence the IR bias of mice undergoing allergic sensitization to Ova. The greatest effect was observed in TM and TH groups, which had an anti-allergic type 1-biased phenotype as measured by isotype-specific antibody (IgG1 and lgE) activity, relatively increased type 1-associated cytokine production (IL-12), as well as high proportion of circulating T-reg cells. Given this, it was found that mice treated with TL were more susceptible to developing clinical allergy than those treated with
TM or TH. Furthermore, TH prevention group was more protective against allergy based on most of the biomarkers measured such as the decrease in specific IgG response in addition to other balance IR parameters. Thus, of all the three prevention groups, the IMD$\mathrm{TH}$ treated mice displayed an increased ability to respond to Ova in terms of antibody activity (decreased specific $\lg G, \lg G 1$, and $\lg \mathrm{E}$ ) and had more balanced cytokine profiles than mice otherwise treated. In order to elucidate mechanisms of anti-allergic response of IMD, we focused on Foxp3 + Treg (CD4+ CD25+ Foxp3+T) cells. The expression of Foxp3 in mouse treated with TM and TH was increased in mice, suggesting that Treg cell polarization was promoted by IMD.

Oral immunotherapy (OIT) is a promising therapeutic approach to treat food allergic patients, however, it is generally considered as medication. Nutritional interventions may provide a new insight to improve the efficacy of OIT for food allergic patients. Dietary non-digestible oligosaccharides mimic the immunomodulatory effects exerted by human milk oligosaccharides in breast-fed infants and have been shown to reduce the risk of developing allergic diseases. ${ }^{25}$ It is also reported that digestible oligosaccharides can cross the intestinal epithelial barrier and directly affect immune cells involved in the process of oral tolerance induction. ${ }^{26,27}$ The capacity of non-digestible oligosaccharides to induce IR modulation and suppress allergic reactions in murine food allergy models suggests they may provide a potential benefit in combination with OIT strategies. ${ }^{28,29}$ It has been reported that oligosaccharide prebiotics have been shown to have a protective effect against allergic manifestations in high risk infants. ${ }^{30}$ Earlier studies have also described that IMD was digested partially only by small intestinal mucosal enzymes, and maltase and isomaltose activities were weakly inhibited. ${ }^{22}$ In this study the effect of IMD could only partially reduce clinical allergic signs, however there 


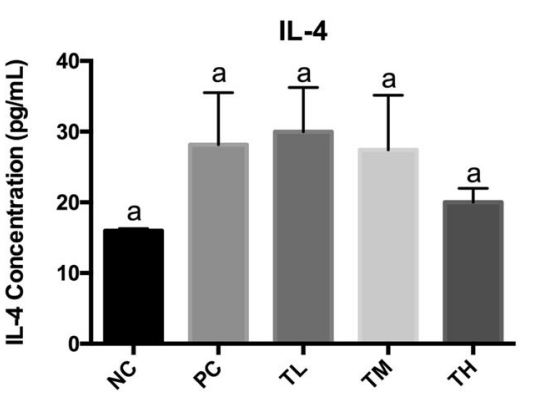

IL-12

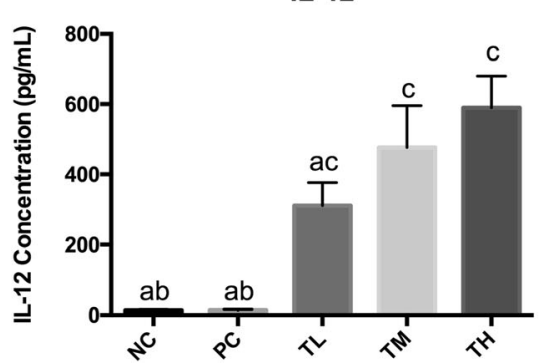

IFN-Y

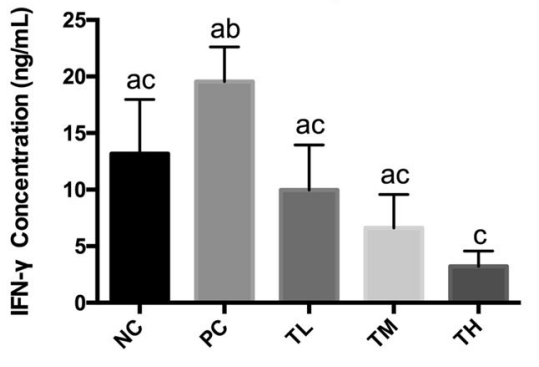

IL-10

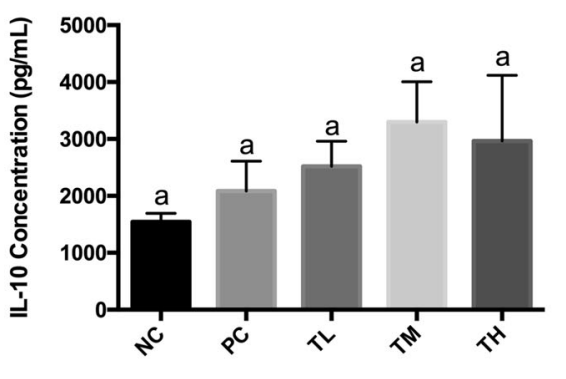

TGF- $\beta$



IL-17



Fig. 6 Cytokine concentration. Spleen was collected from each mouse and pooled within groups $(n=6)$ and splenocytes were isolated and cultured at $2.5 \times 10^{6}$ cells $/ \mathrm{mL}$ concentration of cells in triplicate wells as unstimulated (control) or stimulated with $50 \mu \mathrm{g} /$ well of Ova for $72 \mathrm{~h}$. The cytokine concentration of cell culture supernatants for IL-4, IFN- $\gamma$, IL-12, IL-10, TGF- $\beta$, and IL-17 were determined by ELISA. Different letters indicate significant difference between groups for each cytokine. Significance was taken at $p \leq 0.05$

was a skewed immune response from a type 2 allergic response to a type 1 pro-inflammatory response by the $\mathrm{TH}$ group. These effects could be attributed to the dosage and to the digestibility of IMD by the intestinal mucosal enzymes.

Also, IMD has been confirmed to stabilize micron sized micelles in a manner similar to that of resistant maltodextrin, suggesting the inhibitory effects of IMD on the progression of micronization of micelles. ${ }^{31}$ Adhesion of IMD on the particle surface may have caused an increase in the surface potential. ${ }^{32}$ This could also be attributed to the mechanism of inhibition of allergic response in this study that may have been based on the increase on the surface potential and the dose of IMD used. Thus, it could be concluded that mice treated with TL and TM were comparatively more susceptible to developing clinical allergy than those treated with $\mathrm{TH}$ group which was inclined towards protecting against allergy. Hence in this study, the high dosage of IMD could be a major factor in influencing the suppression of allergic IR. The activity of IMD could also be related to the extent and mechanism of its degradation in the gut that may have influenced the suppression of the allergic response. Also, IMD is resistant to enzymatic hydrolysis, allowing it to pass into the large intestine.

Prebiotic stimulates the growth of protective commensal microbes in the gut significantly changed in infancy fecal microbiota which are associated with the development of food allergy. ${ }^{33}$ The proportion of abundant Bacteroidetes, Proteobacteria, and Actinobacteria phyla were significantly reduced, while the Firmicutes phylum was highly enriched in the food allergy group. $^{34,35} \mathrm{~A}$ growing body of evidence suggests that gut microbiota plays an essential role in gut health and promoting local and systemic immunity. In food allergy children compare to healthy subjects, different levels of short chain fatty acids (SCFAs), in particular of butyrate, have been described. ${ }^{36,37}$ Thus, it is also necessary studying the effect of IMD on microbiota change, SCFAs or of other microbiota-derived metabolites production that could prevent food allergy and modulate immune system in future work. It is well known that IMD is water soluble and directly extracted from plants or made from starch and is generally regarded as safe. ${ }^{27}$ Hence using IMD as a prophylactic candidate for curing egg allergy is a promising approach.
In conclusion, these data provide evidence for the role of IMD in prevention of OVA allergic response in mice by inducing immune tolerance through several ways that includes a Th2-skewed to a Th1-skewed response, a regulatory response involving the transcription factor Foxp3, induction of an increase IL-12 response, and influencing mast cell functionality (suppression of histamine and MMCPT-1). This may suggest that IMD could be a potentially useful candidate for the design of a functional prebiotic food component in targeting management of food allergy.

\section{METHODS}

Experimental design-mice and sensitization

A total of 60 mice ( 12 per group) were used. The mice were housed and maintained under normal husbandry conditions at the Central Animal Facility (University of Guelph). All experimental protocols were in accordance with the Canadian Council for Animal Care guidelines and all animal use protocols were approved by University of Guelph Animal Care Committee (AUP 1567).

\section{Pre-treatments and sensitization}

Figure 8 summarizes the pre-treatment, sensitization, sampling, and challenge schedule. Groups of mice (12 per group) were assigned to one of three prevention groups (IMD in drinking water (1.0, 2.5\%, and $5.0 \% \mathrm{w} / \mathrm{v}$ ) were administered ad lib for 6 weeks) prior to sensitization and continued during sensitization period. Group A was treated with phosphate-buffered saline (PBS; negative control). Ovalbumin (Ova: $50 \mu \mathrm{g} /$ mice) was given by intraperitoneal injection (IP) (dissolved in $50 \mu \mathrm{L}$ of saline and $50 \mu \mathrm{L}$ of aluminum hydroxide gel adjuvant; Sigma-Aldrich, St Louis, MO) at weeks 7,8 , and 9 . Blood was taken on week 12 to measure specific $\mathrm{lgE}$ as an indication of allergic sensitization and confirmed its sensitization (data not shown). Mice were allowed to fast overnight for $8 \mathrm{~h}$ prior to oral challenge on week 13 with $20 \mathrm{mg}$ of Ova given orally via gavage. All mice were humanely euthanized after 30 min of monitoring for allergic signs. Blood was collected for various biomarker assays such as measurement of histamine, mast cell protease, antibody activity and flow cytometry analysis. Spleen was collected for measuring cytokines. 
NC

$\mathrm{A} 02 \mathrm{NC}-1-2$

ㄱ. Gate: (R1 in all) and (M3 in all) and (M4

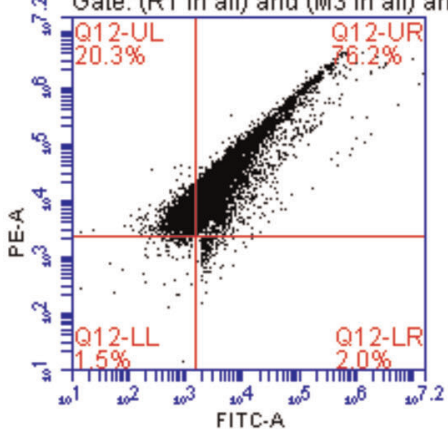

TL

Suv ' Gate: (R1 in all) and (M11 in all) and (N

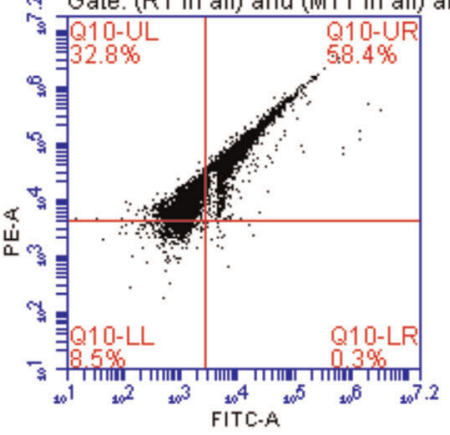

PC

$\mathrm{A} 04 \mathrm{PC}-1-2$

Cy Gate: $R 1$ and (M7 in R1) and (M8 in ( $R^{\circ}$



TM

B02 TM-1-6

Gate: (R1 in all) and (M15 in all) and (I

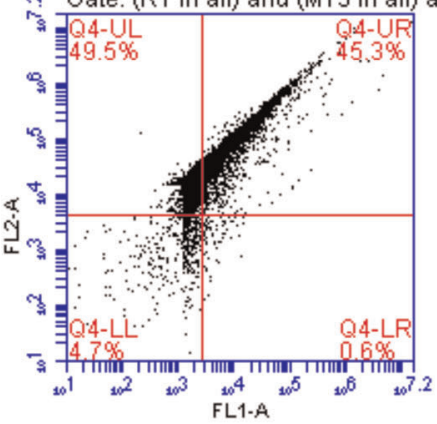

TH

B03 TH-1-5

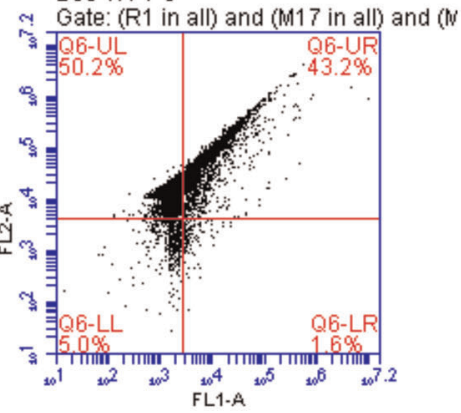

Fig. 7 Flow cytometry. A representative image of CD25+ Foxp3+ cells for each group is shown. Percentage of CD25 + Foxp3 + cells were determined by FACS from whole blood of mice collected at the end of the experiment. Different letters indicate statistically significant differences between groups at $p<0.05$

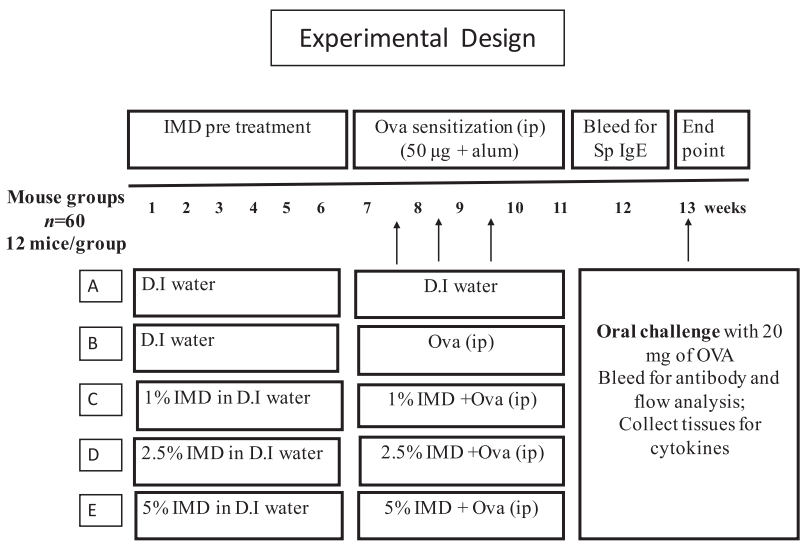

Fig. 8 Pre-treatment and sensitization protocol. Five groups of mice, each group containing of 12 mice were used in this study. Three groups of mice were pre-treated with three different doses of IMD $(1 \%, 2.5 \%$, and $5 \%)$ given orally gavage for the first 6 weeks and continuously administered during sensitization (week 7-11). The negative and positive control group received just water. Mice were sensitized with $50 \mu \mathrm{g}$ of ovalbumin (Ova) given intraperitoneally three times (i.p.). Negative control group did not receive any injection. On week 13 all mice were fasted overnight and challenged with $20 \mathrm{mg}$ Ova and observed for at least $30 \mathrm{~min}$ for clinical signs of allergy and assigned clinical scores. Blood was taken to measure immunoglobulin isotype-associated antibody activity by enzymelinked immunosorbent assay (ELISA) and to isolate blood mononuclear cells for culture and quantification of cytokine concentrations and to measure the proportion of circulating T-regulatory cells (T-regs) by flow cytometry

\section{Clinical signs}

Allergic clinical signs were evaluated in a blinded fashion by four experienced independent observers and scores were assigned as described earlier. ${ }^{24}$ In order to be classified as allergic, mice had to exhibit clinical signs such as scratching, lethargy, gastrointestinal signs (diarrhea and bloody stool) and respiratory difficulty had to occur in combination with one of the aforementioned signs to be scored as a sign and were not themselves sufficient to identify a mouse as allergic. Thus, signs were additive, and clinical score was indicative of the severity of the allergic reaction.

Histamine and mouse mast cell protease assay

Histamine concentrations in serum were determined by Enzyme-Linked Immunosorbent Assay (ELISA) using a commercial kit (Histamine EIA, LDN Labor Diagnostika Nord, Nordhon, Germany) as described by the manufacturer. The mouse mast cell protease enzyme (MMCP) concentration was quantified by ELISA as per manufacturer's recommendation (eBiosciences, California, USA).

Measurement of immunoglobulin isotype-specific antibody activity to Ova by enzyme-linked immunosorbent assay

Sera obtained on week 13 and stored at $-80^{\circ} \mathrm{C}$ were used to determine the $\mathrm{lg}$ isotype-specific antibody activity to Ova by enzyme-linked immunosorbent assay (ELISA). Determination of individual subclasses of serum allergen-specific $\lg E, \lg G, \lg G 1, \lg G 2 \mathrm{a}$, and feces sp-lgA were examined. Optimal antigen-coating conditions were determined. Briefly, 96-well microtiter plates (Costar, Corning Inc., NY, USA) were coated with $2 \mu \mathrm{g} /$ well of Ova (in $100 \mu \mathrm{L}$ of $50 \mathrm{mM}$ carbonate buffer; $\mathrm{NaHCO}_{3} / \mathrm{Na}_{2} \mathrm{CO}_{3}$, $\mathrm{pH}$ 9.6) for $24 \mathrm{~h}$ at $4{ }^{\circ} \mathrm{C}$. Rabbit anti-mouse IgE conjugated to alkaline phosphatase was used for the detection of $\mathrm{lgE}$, and rat monoclonal antimouse IgA was used for IgA followed by streptavidin-HRP conjugate. For 
$\lg G 1$ and $\lg G 2 a$ rat monoclonal anti-mouse $\lg G 1$ and $\lg G 2 a$ were used followed by streptavidin-HRP conjugate. Plates were washed 3 times with $200 \mu \mathrm{L}$ of $0.05 \%$ Tween PBS (PBST $0.01 \mathrm{M}, \mathrm{pH}$ 7.4) per well) after coating with $2 \%$ bovine serum albumin (BSA)/phosphate buffered saline (PBS) at $37^{\circ} \mathrm{C}$ for $1 \mathrm{~h}$. Washing was repeated and sera diluted (1:10, 1:500 for IgE and IgG measurements, 1:100 for IgG1 and IgG2a, no dilution of $\lg \mathrm{A}$ ) in $0.05 \%$ PBST were added at $100 \mu \mathrm{L}$ per well and incubated for overnight at room temperature with gentle shaking. For IgA, proteins were extracted using appropriate inhibitor cocktail ( $1 \mathrm{mM}$ phenylmethanesulfonyl fluoride (PMSF), $0.1 \mathrm{mM}$ ethylenediaminetetraacetic acid (EDTA), $10 \mu \mathrm{g} / \mathrm{mL}$ aprotinin, $10 \mu \mathrm{g} / \mathrm{mL}$ leupeptin and $10 \mu \mathrm{g} / \mathrm{mL}$ pepstatin A; Sigma-Aldrich, St Louis, MO) from feces and protein concentrations were measured according to bicinchonimic assay (BCA) assay. Plates were washed again and the bound antibodies were detected by adding respective detection antibodies at room temperature for $1 \mathrm{~h}$. Specific binding activity was detected by addition of $100 \mu \mathrm{L} /$ well of $3,3^{\prime} 5,5^{\prime}-$ tetramethylbenzidine substrate (Sigma-Aldrich). The reaction was terminated with $1 \mathrm{~N} \mathrm{H}_{2} \mathrm{SO}_{4}$ after $30 \mathrm{~min}$ and absorbance at $450 \mathrm{~nm}$ was measured by a micro titer reader (Bio-Rad 550, Hercules, USA). For total antibody similar sandwich ELISA method was used but the plates were coated by the capture antibody for each isotype.

\section{Cytokine analysis from mouse spleen}

Individual spleens were collected at the end point post challenge aseptically from each mouse single cell suspensions were prepared. Splenocyte cell viability was assessed by trypan blue exclusion. ${ }^{38}$ Cells were cultured in 24 well plates in triplicate at a density of $2.5 \times 10^{6} \mathrm{cells} / \mathrm{mL}$ as unstimulated (control) or stimulated with $50 \mu \mathrm{g} / \mathrm{well}$ of Ova. Culture supernatants were collected after $72 \mathrm{~h}$ incubation at $37^{\circ} \mathrm{C}$ and stored in aliquots at $-20^{\circ} \mathrm{C}$. Concentrations of cytokines were measured in culture supernatants using ELISA cytokine kits following manufacturer's instructions (ebiosciences, CA, USA for IL-4, IFN- $\gamma$, IL-10, IL-12, IL-17A, and TGF- $\beta$ ).

Determination of proportion of T-regulatory cells in lymphocytes of whole blood by flow cytometry

Blood collected on week 13 was also used to measure the proportion of blood T-regs from all mice by flow cytometry, based on CD25 and forkhead box P3 (Foxp3) positivity. One hundred microliters of blood from each mouse was used for double staining with anti-mouse CD25 (558642; BD biosciences, California, USA) and R-phycoerythrin (PE)-conjugated antimouse Foxp3 (eBiosciences, San Diego, CA), Analysis of labeled cells was performed using an Accuri Flow Cytometer (BD). Isotype and unstained controls were included to confirm specific staining of anti-CD25 and antiFoxp3 and to adjust compensation. The antibody isotype controls used were mouse lgG1 negative control for $\mathrm{CD} 25$ and $\mathrm{PE}$-conjugated rat lgG2a for Foxp3. Unstained samples were treated with wash buffer, PBS with $0.1 \%$ bovine serum albumin (PBA). The staining protocol was used as per the manufacturer's instruction for whole blood sample (BD Bioscience). Live cells were gated based on forward and $90^{\circ}$ light scatter characteristics. At least 10,000 events were acquired from each sample and all data sets were analyzed using Accuri Express software (BD Bioscience).

\section{Statistical analysis}

Statistical calculations were performed using the GraphPad Prism 5.0 package (GraphPad Software, San Diego, CA, USA). All data were expressed as means \pm SEM and subjected to ANOVA analyses followed by post hoc multiple comparison using Tukey's test. Comparison of all the endpoint differences with a level of $P<0.05$ was considered significant.

\section{Reporting summary}

Further information on experimental design is available in the Nature Research Reporting Summary linked to this paper.

\section{DATA AVAILABILITY}

The datasets generated during and/or analyzed during the current study are available from the corresponding author on reasonable request.

Received: 5 October 2018; Accepted: 23 August 2019; Published online: 06 November 2019

\section{REFERENCES}

1. Boyce, J. A. et al. Guidelines for the diagnosis and management of food allergy in the United States: report of the NIAID-sponsored expert panel. J. Allergy Clin. Immunol. 126, 1-58 (2010).

2. Gupta, R. S. et al. The prevalence, severity, and distribution of childhood food allergy in the United States. Pediatrics 128, 9-17 (2011).

3. Onyinye, I. et al. Food allergy. Curr. Gastroenterol. Rep. 20, 17 (2018).

4. Lianne, S. et al. Adjusting for nonresponse bias corrects overestimates of food allergy prevalence. J. Allergy Clin. Immunol. 3, 291-293 (2015).

5. Keet, C. A. et al. Temporal trends and racial/ethnic disparity in self-reported pediatric food allergy in the United States. Ann. Allergy Asthma Immunol. 112, 222-229 (2014)

6. Kamdar, T. A. et al. Prevalence and characteristics of adult-onset food allergy. J. Allergy Clin. Immunol. Pract. 3, 114-115 (2015).

7. Sampson, H. A. Food allergy: past, present and future. Allergol. Int. 65, 363-369 (2016).

8. Lanser, B. J., Wright, B. L., Orgel, K. A., Vickery, B. P. \& Fleischer, D. M. Current options for the treatment of food allergy. Pediatr. Clin. 62, 1531-1549 (2015).

9. Houben, G. P. et al. Prioritisation of allergenic foods with respect to public health relevance: report from an ILSI Europe food allergy task force expert group. Food Chem. Tox. 89, 8-18 (2016).

10. Wood, R. A. Food allergen immunotherapy: current status and prospects for the future. J. Allergy Clin. Immunol. 137, 973-982 (2016).

11. Caubet, J. C. \& Wang, J. Current understanding of egg allergy. Pediatr. Clin. 58, 427-443 (2011).

12. Heine, R. G., Laske, N. \& Hill, D. J. The diagnosis and management of egg allergy. Curr. Allergy Asthma Rep. 6, 145-152 (2006).

13. Järvinen, K. M. et al. Specificity of IgE antibodies to sequential epitopes of hen's egg ovomucoid as a marker for persistence of egg allergy. Allergy 62, 758-765 (2007).

14. Wang, J. Management of the patient with multiple food allergies. Curr. Allergy Asthma Rep. 10, 171-277 (2010).

15. Tsuda, M. et al. Dietary fructo-oligosaccharides attenuate early activation of CD4+ T cells which produce both Th1 and Th2 cytokines in the intestinal lymphoid tissues of a murine food allergy model. Int. Arch. Allergy Immunol. 174, 121-132 (2017).

16. Gibson, G. R., Hutkins, R. \& Cani, P. D. Expert consensus document: The International Scientific Association for Probiotics and Prebiotics (ISAPP) consensus statement on the definition and scope of prebiotics. Nat. Rev. Gastroenterol. Hepatol. 14, 491 (2017)

17. Tsusaki, K. et al. Structure of a novel highly branched a-glucan enzymatically produced from maltodextrin. Carbohydr. Res. 344, 2151-2156 (2009).

18. Tsusaki, K. et al. Purification and characterization of highly branched a glucan-producing enzymes from Paenibacillus sp. PP710. Biosci. Biotechnol. Biochem. 76, 721-731 (2012).

19. Chow, J. M. Probiotics and prebiotics: a brief overview. J. Ren. Nutr. 12, 76-86 (2002).

20. Moshfegh, A. J. et al. Presence of inulin and oligofructose in the diets of Americans. J. Nutr. 129, 1407-1411 (1999).

21. Okuma, K. What is resistant maltodextrin?. Process. Struct. Prop. Diet. Fiber $\mathbf{8 8}$ 151-160 (2008)

22. Sadakiyo, T. et al. Attenuation of postprandial blood glucose in humans consuming isomaltodextrin: carbohydrate loading studies. Food Nutr. Res. 61, 1-10 (2017).

23. Majumder, K. et al. Intervention of isomaltodextrin mitigates intestinal inflammation in a dextran sodium sulfate-induced mouse model of colitis via inhibition of toll-like receptor-4. J. Agric. Food Chem. 65, 810-817 (2017).

24. Rupa, P., Nakamura, S., Katayama, S. \& Mine, Y. Effects of ovalbumin glycoconjugates on alleviation of orally induced egg allergy in mice via dendritic-cell maturation and T-cell activation. Mol. Nutr. Food Res. 58, 405-417 (2014).

25. Jeurink, P. V. et al. Mechnisms underlying immune effects of dietary oiligosaccharides. Am. J. Clin. Nutr. 98, 572S-777S (2013).

26. Eiwegger, $\mathrm{T}$. et al. Oligosaccharides: in vitro evidence for gastrointestinal epithelial transfer and immunomodulatory properties. Pediatr. Allergy Immunol. 21, 1179-1188 (2010).

27. Nauta, A. J. \& Garssen, J. Evidence-based benefitrs of specific mixtures of nondigestible oligosaccharides on the immune system. Carbohydr. Polym. 95 263-265 (2013).

28. Wagenaar, L. et al. Dietary Supplementation with nondigestible oligosaccharides reduces allergic symptoms and supports low dose oral immunotherapy in a oeanut allergy mouse model. Mol. Nutr. Food Res. e1800369. https://doi.org/ 10.1002/mnfr.201800369. (2018).

29. Hayen, S. M. et al. Non-digestible oligosaccharides can Suppress basophil degranulation in whole blood of peanut-allergic patients. Front Immunol 9, 1265 https://doi.org/10.3389/fimmu.2018.01265 (2018). 
30. Arslanoglu, S. et al. Early neutral prebiotic oligosaccharide supplentation reduces the incidence of some allergic manifestations in the first 5 years of life. J. Biol. Regul. Homeost. Agents 26, 49-59 (2012).

31. Kishimoto, Y. et al. Effect of resistant maltodextrin on digestion and absorption of lipids. J. Health Sci. 55, 838-844 (2009).

32. Hanaor, D. A. et al. Scalable surface area characterization by electrokinetic analysis of complex anion adsorption. Langmuir 30, 15143-15152 (2014).

33. Gourbeyre, P., Denery, S. \& Bodinier, M. Probiotics and synbiotics: impact on the gut immune system and allergic reactions. J. Leukoc. Biol. 89, 685-695 (2011).

34. Abbring, S. et al. Post sensitization administration of non-digestible oiligosaccharides and Bifidobacterium breve $\mathrm{M}-16 \mathrm{~V}$ redcues allergic symotoms in mice. Immune Inflamm. Dis. 4, 155-165 (2016).

35. Kerperien, J. et al. Non-digestible oiligosaccharides modulate intestinal immune activation and suppress cow's milk allergic symptoms. Pediatr. Allergy Immunol. 25, 745-754 (2014).

36. Ling, Z. et al. Altered fecal microbiota composition associated with food allergy in infants. Appl Environ. Microbiol. 80, 2546-2554 (2014).

37. Berni Canani, R. et al. Gut microbiota composition and butyrate production in children affected by non-lgE-mediated cow's milk allergy. Sci. Rep. 8, 12500, https://doi.org/10.1038/s41598-018-30428-3 (2018).

38. Louis, K. S. \& Siegel, A. C. Cell viability analysis using trypan blue: manual and automated methods. In Mammalian Cell Viability: Methods and Protocols, Methods in Molecular Biology. Vol. 740 (ed. Martin J. Stoddart) 7-12 (Springer Science, Berlin, 2011).

\section{ACKNOWLEDGEMENTS}

The authors would like to acknowledge funding support to Y. Mine from NSERC Discovery grant. The authors would like to thank all the staff at the Central Animal facility, University of Guelph and lab colleagues for their skillful technical assistance in help with the animal study.

\section{AUTHOR CONTRIBUTIONS}

Y.M., K.M., P.R. and H.W. designed the study and wrote the paper. Y.J. and K.M. contributed to data collection. Y.M. and K.M. performed the statistical analysis and interpretation of the results. Y.M., T.S., Y.T., R.T. and H.M. performed proof reading. All authors read and approved the final paper.

\section{COMPETING INTERESTS}

The authors declare no competing interests.

\section{ADDITIONAL INFORMATION}

Supplementary information is available for this paper at https://doi.org/10.1038/ s41538-019-0057-5.

Correspondence and requests for materials should be addressed to Y.M.

Reprints and permission information is available at http://www.nature.com/ reprints

Publisher's note Springer Nature remains neutral with regard to jurisdictional claims in published maps and institutional affiliations.

Open Access This article is licensed under a Creative Commons Attribution 4.0 International License, which permits use, sharing, adaptation, distribution and reproduction in any medium or format, as long as you give appropriate credit to the original author(s) and the source, provide a link to the Creative Commons license, and indicate if changes were made. The images or other third party material in this article are included in the article's Creative Commons license, unless indicated otherwise in a credit line to the material. If material is not included in the article's Creative Commons license and your intended use is not permitted by statutory regulation or exceeds the permitted use, you will need to obtain permission directly from the copyright holder. To view a copy of this license, visit http://creativecommons. org/licenses/by/4.0/.

(c) The Author(s) 2019 